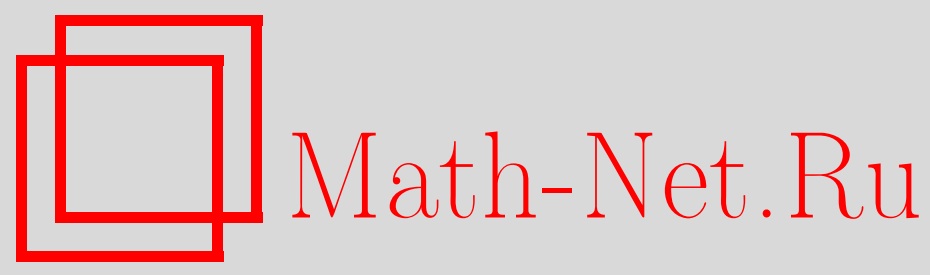

K. О. Бесов, О непрерывности обобщенного оператора Немыцкого в пространствах дифференцируемых функций, матем. заметки, 2002, том 71, выпуск 2, 168-181

DOI: https://doi.org/10.4213/mzm337

Использование Общероссийского математического портала Math-Net.Ru подразумевает, что вы прочитали и согласны с пользовательским соглашением http://www.mathnet.ru/rus/agreement

Параметры загрузки:

IP: 3.80 .181 .102

26 апреля 2023 г., 14:03:59

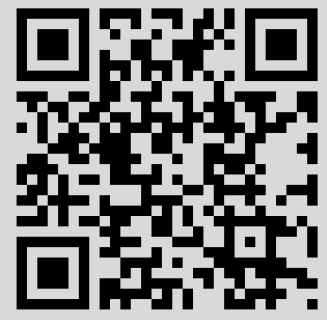




\title{
О НЕПРЕРЫВНОСТИ ОБОБЩЕННОГО OПЕРАТОРА НЕМЫЦКОГО В ПРОСТРАНСТВАХ ДИФФЕРЕНЦИРУЕМЫХ ФУНКЦИЙ
}

\author{
К. О. Бесов
}

Получены достаточные условия непрерывности общего нелинейного оператора суперпозиции (обобщенного оператора Немыцкого), действующего из пространства $C^{m}(\bar{\Omega})$ дифференцируемых функций на ограниченной области $\Omega$ в лебегово пространство $L_{p}(\Omega)$. При этом рассматриваются операторы, значения которых на функции $u \in C^{m}(\bar{\Omega})$ локально определяются значениями как самой функции $u$, так и всех ее частных производных до порядка $m$ включительно. Показано, что в некоторых частных случаях полученные достаточные условия являются и необходимыми. Применение результатов проиллюстрировано на конкретных примерах и, кроме того, даны приложения к теории пространств Соболева.

Библиограбиия: 8 названий.

1. Введение. Пусть $\Omega \subset \mathbb{R}^{n}$ - ограниченная область, $f$ - функция двух переменных $x, u, x \in \Omega, u \in \mathbb{R}$, удовлетворяющая условию Каратеодори, т.е. $f$ измерима по $x$ при любом $u \in \mathbb{R}$ и непрерывна по $u$ при почти всех $x \in \Omega$. Оператором Немыикого называется отображение $\boldsymbol{f}$, переводящее (измеримую) функцию $u(x)$ в функцию $\boldsymbol{f} u(x)=f(x, u(x)), x \in \Omega$ (которая также будет измеримой в силу условия Каратеодори).

Хорошо известно следующее утверждение, полученное в работах Красносельского и Вайнберга (см., например, [1], [2]).

Tеорема А. Пусть оператор $f$ действует из $L_{q}(\Omega)$ в $L_{p}(\Omega), 1 \leqslant q, p<\infty$ (m.e. для любой $u \in L_{q}(\Omega)$ ее образ $\boldsymbol{f}$ и лежит в $L_{p}(\Omega)$ ). Тогда оператор $\boldsymbol{f}$ непрерывен, ограничен (т.е. переводит ограниченные мнохества в ограниченные) и найдутся функиия $a \in L_{p}(\Omega)$ иконстанта $b>0$ такие, что

$$
|f(x, u)| \leqslant a(x)+b|u|^{q / p}, \quad x \in \Omega, \quad u \in \mathbb{R} .
$$

Обратно, если при некоторых $а \in L_{p}(\Omega)$ и $b>0$ выполнено (1), то оператор $f$ действует из $L_{q}(\Omega)$ в $L_{p}(\Omega)$, непрерывен и ограничен.

Работа выполнена при финансовой поддержке Российского фонда фундаментальных исследований, гранты № 99-01-00045 и № 01-01-06288, программы "Ведущие научные шкколы", грант № 0015-96047, и фонда INTAS, грант № 00-136. 
Этот результат без труда переносится на вектор-функции $u=\left(u_{1}, \ldots, u_{m}\right)$ с компонентами $u_{j} \in L_{q_{j}}$ и функции $f: \Omega \times \mathbb{R}^{m} \rightarrow \mathbb{R}$. В этом случае неравенство (1) переходит в

$$
|f(x, u)| \leqslant a(x)+b \sum_{j=1}^{m}\left|u_{j}\right|^{q_{j} / p}, \quad x \in \Omega, \quad u \in \mathbb{R}^{m} .
$$

Теорема А была доказана в 1950-х гг. Позднее Шрагин получил необходимые и достаточные условия для действия оператора $f$ из пространства $C(\bar{\Omega})$ непрерывных на замькании области функций в $L_{p}(\Omega)$.

Tеорема В (см. [3]). Пусть оператор $f$ действует из $C(\bar{\Omega})$ в $L_{p}(\Omega), p<\infty$. Тогда оператор $f$ равномерно непрерывен на каждом ограниченном множестве функиий из $C(\bar{\Omega})$ (в частности, непрерывен и ограничен) $и$

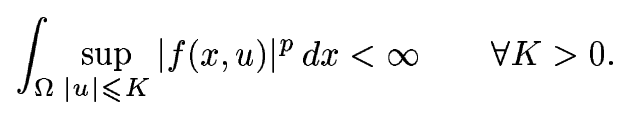

Обратно, если выполнено (2), то оператор $f$ действует из $C(\bar{\Omega})$ в $L_{p}(\Omega)$ равномерно непрерывно на каждом ограниченном множестве из $C(\bar{\Omega})$.

Теорема В также остается справедливой для вектор-функций $u$. С другой стороны, условие (2) может быть переписано в более простом виде, которьй чаще используется в приложениях:

$$
|f(x, u)| \leqslant a(x) b(u), \quad x \in \Omega, \quad u \in \mathbb{R},
$$

где $a \in L_{p}(\Omega)$ и $b: \mathbb{R} \rightarrow \mathbb{R}$ - непрерьвная функция. Действительно, очевидно, что из (3) вытекает (2). Покажем обратное. Положим $f_{k}(x)=\sup _{|u| \leqslant k}|f(x, u)|$ при $k=1,2, \ldots$; $f_{k} \in L_{p}(\Omega)$ в силу (2). Определим

$$
a(x)=\sum_{k=1}^{\infty} \frac{f_{k}(x)}{2^{k}\left\|f_{k}\right\|_{L_{p}}} .
$$

Тогда $a \in L_{p}(\Omega)$ и

$$
|f(x, u)| \leqslant f_{[|u|]+1}(x) \leqslant 2^{[|u|]+1}\left\|f_{[|u|]+1}\right\|_{L_{p}} a(x) \leqslant a(x) b(u),
$$

где $b(u)$ - произвольная непрерывная мажоранта функции $2^{[|u|]+1}\left\|f_{[|u|]+1}\right\|_{L_{p}}$.

В данной работе нас будет интересовать действие оператора $f$ в более "узких" пространствах $C^{m}(\bar{\Omega}), m \in \mathbb{N}$. Напомним, что по определению функция $u$ принадлежит $C^{m}(\bar{\Omega})$, если она непрерывна на $\bar{\Omega}$, имеет в $\Omega$ все частные производные порядка не выше $m$ и эти производные могут быть непрерывно продолжены на $\bar{\Omega}$. Норма в $C^{m}(\bar{\Omega})$ определяется как $\|u\|_{C^{m}}=\|u\|_{C}+\cdots+\left\|\nabla^{m} u\right\|_{C}$, где $\nabla^{m} u(x)=\left\{D^{\alpha} u(x)|| \alpha \mid=m\right\}$, $D^{\alpha}=\partial^{|\alpha|} /\left(\partial x_{1}^{\alpha_{1}} \cdots \partial x_{n}^{\alpha_{n}}\right),|\alpha|=\alpha_{1}+\cdots+\alpha_{n}, \alpha_{j} \in \mathbb{N}_{0}=\mathbb{N} \cup\{0\}$. 
ПРИМЕР 1. Положим $\Omega=(0,1)$,

$$
f(x, u)=\frac{|u|^{r}}{x^{2}+\left(u^{2}-x\right)^{2}} .
$$

Можно показать, что соответствующий оператор $\boldsymbol{f}$ действует из $C^{1}$ в $L_{1}$ при $r>1$. Если $1<r<2$, то $f$ неограничен (даже на константах); при $r=2$ он ограничен, но не является непрерьвньп, и только при $r>2$ его действие распространяется на все непрерьвные функции.

Нас будет интересовать возможность ослабления условия (3) с сохранением свойства непрерьвности на $C^{m}(\bar{\Omega})$. Из недавних публикаций в этом направлении отметим работы Чиаппинелли и Нугари [4] и Зикеля [5]. В первой из них изучаются операторы Немьщкого, действующие из пространства функций, удовлетворяющих условию Гёльдера, в себя (нас же интересует действие из $C^{m}$ в $L_{p}$ ). Во второй рассмотрен более широкий спектр функциональных пространств, однако исследуются свойства только автономных операторов Немыцкого (т.е. функция $f$ не зависит от $x$ и является функцией одной переменной $u$ ). Более полную библиографию можно найти в монографии [6].

Основное отличие настоящей работы от указанных вьше состоит в том, что мы рассматриваем операторы $\boldsymbol{f}$ более общего вида, а именно

$$
C^{m}(\bar{\Omega}) \ni u \rightarrow \boldsymbol{f} u, \quad \boldsymbol{f} u(x)=f\left(x, u(x), \nabla u(x), \ldots, \nabla^{m} u(x)\right), \quad x \in \Omega,
$$

что также обусловлено приложениями. Функция $f\left(x, \mathscr{U}_{m}\right)$ всюду предполагается каратеодориевой, т.е. непрерывной по совокупности переменных $\mathscr{U}_{m}=\left\{u_{\alpha}|| \alpha \mid \leqslant m\right\}$ при почти всех $x$ и измеримой по $x$ при всех $\mathscr{U}_{m}$. В частном случае, когда $f$ не зависит от $\nabla u, \ldots, \nabla^{m} u$, получаем обычный оператор Немьпкого $\boldsymbol{f} u(x)=f(x, u(x))$.

Мы также расширим область значений оператора $f$ с $\mathbb{R}$ до $\overline{\mathbb{R}}=\mathbb{R} \cup\{\infty\}$, наделив последнее множество топологией окружности. Таким образом, если $f\left(x_{0}, u_{0}\right)=\infty$, то непрерьвность по $u$ означает, что $\left|f\left(x_{0}, u\right)\right| \rightarrow \infty$ при $u \rightarrow u_{0}$. Это расширение не дает ничего нового, если рассматривать оператор $\boldsymbol{f}$ в пространстве непрерьвных функций, однако, как показывает следующий пример, уже для $C^{1}(\bar{\Omega})$ ситуация будет иной.

ПримеР 2. Положим $\Omega=(0,1), r, r^{\prime}>0$,

$$
f\left(x, u, u^{\prime}\right)=\frac{1}{|u|^{r}+\left|u^{\prime}-1\right|^{r^{\prime}}} .
$$

В этом случае оператор $f$ действует из $C^{1}$ в $L_{p}$ при $r<1 / p$ и любом $r^{\prime}$. Ниже мы покажем (теорема 2), что он непрерывен, но не является ограниченньм. При этом верхняя грань, аналогичная той, что фигурирует в (2), обращается в бесконечность в каждой точке $x$.

Примеры 1 и 2 показьвают независимость свойств непрерьвности и ограниченности оператора $\boldsymbol{f}$ на пространствах дифференцируемых функций. Это одно из существенных отличий от действия на непрерьвных функциях, поэтому рассчитывать на получение необходимых и достаточных условий, аналогичных (3), здесь уже не приходится. Мы сосредоточим свое внимание на достаточных условиях непрерьвности. В п. 2 мы формулируем одно общее достаточное условие непрерьвности (теорема 1) и приводим соответствующее доказательство. Однако это условие не всегда можно легко проверить в 
конкретной ситуации. Поэтому в п. 3 мы рассматриваем частный случай операторов $\boldsymbol{f}$, для которых соответствующая функция $f\left(x, \mathscr{U}_{m}\right) \equiv f\left(\mathscr{U}_{m}\right)$ не зависит от $x$ - их можно назвать автономными. Полученное нами в этом случае достаточное условие имеет более наглядный вид, чем условие из п. 2 для операторов Немьщкого общего вида. Для пространства $C^{1}$ это условие является также необходимым, а при $m \geqslant 2$ оно, хотя и решает главную задачу - ослабление условия (3), все же достаточно далеко от необходимого. В заключительном пункте в качестве следствия теоремы 1 получено достаточное условие непрерывности для операторов еще одного частного вида, а также рассмотрены приложения к нелинейным дифференциальным уравнениям с частными производными.

2. Достаточные условия непрерывности. Напомним, что область $\Omega$ всюду предполагается ограниченной. Для измеримой функции $u$ и $\delta>0$ положим

$$
\omega_{p}(u, \delta)=\sup _{A \subset \Omega,|A| \leqslant \delta} \int_{A}|u|^{p}
$$

где $|A|$ - лебегова мера (измеримого) множества $A$. Если $u \in L_{p}(\Omega)$, то в силу абсолютной непрерывности интеграла $\omega_{p}(u, \delta) \rightarrow 0$ при $\delta \rightarrow 0$.

ОПРЕДЕЛЕНИЕ 1. Будем говорить, что оператор $f: C^{m}(\bar{\Omega}) \rightarrow L_{p}(\Omega)$ обладает свойством (UC) на некотором множестве $E \subset C^{m}(\bar{\Omega})$, если

$$
\sup _{u \in E} \omega_{p}(\boldsymbol{f} u, \delta) \rightarrow 0, \quad \delta \rightarrow 0
$$

Соотношение (5) означает, что образы точек из $E$ под действием оператора $\boldsymbol{f}$, возведенные в $p$-ю степень, имеют равномерно абсолютно непрерывные интегралы.

Скажем, что оператор $\boldsymbol{f}$ обладает свойством (С) в точке $u \in C^{m}(\bar{\Omega})$, если он обладает свойством (UC) в некоторой окрестности этой точки.

Следующий критерий непрерывности оператора $\boldsymbol{f}$ справедлив не только для пространств $C^{m}$, но и для любых функциональных пространств, сходимость в которых влечет сходимость по мере.

УТВЕРЖДЕНИЕ 1. Оператор $f: C^{m}(\bar{\Omega}) \rightarrow L_{p}(\Omega)$ непрерывен в точке $u_{0}$ тогда и только тогда, когда

$$
\lim _{\varepsilon \rightarrow 0, \delta \rightarrow 0} \sup _{\left\|u-u_{0}\right\|_{C}} \omega_{p}(\boldsymbol{f} u, \delta)=0
$$

ДокАЗАТЕЛьСтво. Пусть оператор $f$ непрерывен в точке $u_{0}$. Имеем

$\omega_{p}(\boldsymbol{f} u, \delta) \leqslant 2^{p-1}\left[\omega_{p}\left(\boldsymbol{f} u-\boldsymbol{f} u_{0}, \delta\right)+\omega_{p}\left(\boldsymbol{f} u_{0}, \delta\right)\right] \leqslant 2^{p-1} \int_{\Omega}\left|\boldsymbol{f} u-\boldsymbol{f} u_{0}\right|^{p}+2^{p-1} \omega_{p}\left(\boldsymbol{f} u_{0}, \delta\right)$.

Первое слагаемое в правой части стремится к нулю при $u \rightarrow u_{0}$ в силу непрерьвности оператора, а второе есть $o(1)$ при $\delta \rightarrow 0$ в силу абсолютной непрерьвности интеграла. 
Обратно, пусть выполнено (6). Из сходимости $u_{n} \rightarrow u_{0}$ в $C^{m}$ вытекает сходимость по мере. Но тогда, как известно (см., например, [1]), $\boldsymbol{f} u_{n}$ сходится к $\boldsymbol{f} u_{0}$ по мере. Для произвольного $\gamma>0$ имеем

$$
\begin{aligned}
\int_{\Omega}\left|\boldsymbol{f} u_{n}-\boldsymbol{f} u_{0}\right|^{p} & \leqslant \int_{\left|\boldsymbol{f} u_{n}-\boldsymbol{f} u_{0}\right|<\gamma} \gamma^{p}+2^{p-1} \int_{\left|\boldsymbol{f} u_{n}-\boldsymbol{f} u_{0}\right| \geqslant \gamma}\left[\left|\boldsymbol{f} u_{n}\right|^{p}+\left|\boldsymbol{f} u_{0}\right|^{p}\right] \\
& \leqslant \gamma^{p}|\Omega|+2^{p} \sup _{\left\|u-u_{0}\right\|_{C^{m}} \leqslant \varepsilon_{n}} \omega\left(\boldsymbol{f} u, \delta_{\gamma, n}\right),
\end{aligned}
$$

где $\varepsilon_{n}=\left\|u_{n}-u_{0}\right\|_{C^{m}}$ и $\delta_{\gamma, n}=\left|\left\{x \in \Omega:\left|\boldsymbol{f} u_{n}-\boldsymbol{f} u_{0}\right| \geqslant \gamma\right\}\right|$. За счет выбора $\gamma>0$ первое слагаемое в правой части (7) можно сделать сколь угодно малым. При фиксированном $\gamma$ и $n \rightarrow \infty$ имеем $\varepsilon_{n} \rightarrow 0$ и $\delta_{\gamma, n} \rightarrow 0$. Отсюда в силу (6) получаем $\boldsymbol{f} u_{n} \rightarrow \boldsymbol{f} u_{0}$ в $L_{p}(\Omega)$.

СлЕдСТВИЕ 1. Если оператор $f$ обладает свойством (C) в точке $u_{0} \in C^{m}(\bar{\Omega})$, то он непрерывен в точке $u_{0}$.

Отметим, что обратное утверждение неверно: существуют операторы, непрерывные в каждой точке, но не обладаюшие свойством (C) в одной или нескольких точках. С другой стороны, оператор может обладать свойством $(\mathrm{C})$ в каждой точке, но не обладать свойством (UC) на шаре - таким будет оператор, рассмотренньй нами в примере 2.

Обозначим через $\mathscr{P}_{m}$ совокупность многочленов степени $m$, а через

$$
\mathscr{P}_{m}(K)=\left\{p \in \mathscr{P}_{m}:\|p\|_{C^{m}} \leqslant K\right\}
$$

пересечение $\mathscr{P}_{m}$ с шаром радиуса $K$ пространства $C^{m}(\bar{\Omega})$. Для формулировки основного результата - достаточного условия непрерьвности оператора $\boldsymbol{f}$ на всем $C^{m}(\bar{\Omega})-$ нам потребуется несколько сузить класс рассматриваемых областей.

УСЛовИЕ 1. Каждая функиия $u \in C^{m}(\bar{\Omega})$ может быть некоторым образом продолэсена до функиии $\widetilde{u} \in C^{m}\left(\mathbb{R}^{n}\right)$. При этом $\|\widetilde{u}\|_{C^{m}\left(\mathbb{R}^{n}\right)} \leqslant c_{\Omega}\|u\|_{C^{m}(\bar{\Omega})}$, әде константа $c_{\Omega}$ не зависит от $и$.

Условие 1 выполнено, например, если множество внутренних точек замыкания $\bar{\Omega}$ удовлетворяет сильному условию конуса (см. [7]) или если граница $\partial \bar{\Omega}$ липшицева.

ТЕОрема 1. Пусть выполнено условие 1. Если оператор $f$ обладает свойством (C) в каждой точке $u \in \mathscr{P}_{m}$, или, что то же самое, обладает свойством (UC) в некоторой окрестности множества $\mathscr{P}_{m}(K)$ при любом $K>0$, то он непрерывен на $C^{m}(\bar{\Omega})$.

ДокАЗАТЕЛЬСтво. Прежде всего поясним эквивалентность предположений теоремы 1. Пусть оператор $f$ обладает свойством $(\mathrm{C})$ в каждой точке $u \in \mathscr{P}_{m}$. Тогда он обладает свойством (UC) в некоторой окрестности каждой точки $u \in \mathscr{P}_{m}(K) \subset \mathscr{P}_{m}$. Объединение всех этих окрестностей образует открытое покрытие компактного множества $\mathscr{P}_{m}(K)$. Выделим из него конечное подпокрытие и обозначим объединение точек этого подпокрытия через $E$. Множество $E$ является окрестностью множества $\mathscr{P}_{m}(K)$, в которой оператор $\boldsymbol{f}$ обладает свойством (UC). В силу компактности $E$ содержит $\varepsilon$-окрестность множества $\mathscr{P}_{m}(K)$ при некотором $\varepsilon>0$.

Рассмотрим произвольную точку $u_{0} \in C^{m}(\bar{\Omega})$ и покажем, что в ней оператор $\boldsymbol{f}$ обладает свойством (C). Тогда утверждение теоремы будет вытекать из следствия 1. 
Каждой точке $x \in \bar{\Omega}$ поставим в соответствие многочлен $p_{x} \in \mathscr{P}_{m}$, являющийся многочленом Тейлора степени $m$ для $u_{0}$ в точке $x$. Очевидно, $p_{x}$ непрерывно зависит от $x \in \bar{\Omega}$, а значит, множество $\left\{p_{x}: x \in \bar{\Omega}\right\}$ ограничено в $C^{m}$ и лежит в $\mathscr{P}_{m}(K)$ при некотором $K>0$. Зафиксируем такое $K$ и соответствуюшее ему $\varepsilon$, о котором шла речь в первом абзаце доказательства.

Каждой точке $x \in \bar{\Omega}$ поставим в соответствие открытую вьпуклую окрестность $O_{x} \ni x$ такую, что $\left\|\widetilde{u}_{0}-p_{x}\right\|_{C^{m}}\left(O_{x}\right) \leqslant \varepsilon N^{-1}$, где $\widetilde{u}_{0}-$ продолжение функции $u$ из класса $C^{m}\left(\mathbb{R}^{n}\right)$, а $N$ - достаточно большое положительное число, точное значение которого будет указано ниже. Объединение всех $O_{x}$ образует открытое покрытие компакта $\bar{\Omega}$. Пусть $O_{1}, \ldots, O_{l}$ - некоторое конечное подпокрытие и $p_{1}, \ldots, p_{l}$ - соответствующие многочлены.

Рассмотрим произвольную функцию $u \in C^{m}(\bar{\Omega}),\left\|u-u_{0}\right\|_{C^{m}} \leqslant \varepsilon N^{-1}$. В силу условия 1 ее можно продолжить до функции $\widetilde{u} \in C^{m}\left(\mathbb{R}^{n}\right)$ так, чтобы $\left\|\widetilde{u}-\widetilde{u}_{0}\right\|_{C^{m}} \leqslant c_{\Omega} \varepsilon N^{-1}$ (сначала продолжаем $u-u_{0}$, а затем к результату прибавляем $\widetilde{u}_{0}$ ). Пусть $u_{j}-$ сужение функции $\widetilde{u}$ на $\bar{O}_{j}, j=1, \ldots, l$. Тогда

$$
\left\|p_{j}-u_{j}\right\|_{C^{m}\left(\bar{O}_{j}\right)} \leqslant c_{\Omega} \varepsilon N^{-1}+\varepsilon N^{-1}=\left(c_{\Omega}+1\right) \varepsilon N^{-1}, \quad j=1, \ldots, l .
$$

Нам потребуется следующий факт.

УТВЕРЖДЕНИЕ 2 (см., например, [8]). Пусть $O \subset \mathbb{R}^{n}$ - выпуклое открытое множество. Каждую функиию $u \in C^{m}(\bar{O}), m \in \mathbb{N}$, можно продолжить до функиии $\widetilde{u} \in C^{m}\left(\mathbb{R}^{n}\right)$ так, что $\|u\|_{C^{m}\left(\mathbb{R}^{n}\right)} \leqslant c_{1}\|u\|_{C^{m}(\bar{O})}$, әде константа $c_{1}$ зависит только от $m$ и $n$.

Пусть $\widetilde{u}_{j}$ - такое продолжение функции $u_{j}$ на $\mathbb{R}^{n}$, что

$$
\left\|p_{j}-\widetilde{u}_{j}\right\|_{C^{m}(\bar{\Omega})} \leqslant c_{1}\left(c_{\Omega}+1\right) \varepsilon N^{-1}, \quad j=1, \ldots, l .
$$

Положим $N=c_{1}\left(c_{\Omega}+1\right)$. Тогда каждая из $\widetilde{u}_{j}$ попадает в $\varepsilon$-окрестность множества $\mathscr{P}_{m}(K)$, откуда

$$
\omega_{p}\left(\boldsymbol{f} \widetilde{u}_{j}, \delta\right) \rightarrow 0, \quad \delta \rightarrow 0, \quad j=1, \ldots, l
$$

С другой стороны,

$$
\omega_{p}(\boldsymbol{f} u, \delta) \leqslant \sum_{j=1}^{l} \omega_{p}\left(\boldsymbol{f} \widetilde{u}_{j}, \delta\right) \rightarrow 0, \quad \delta \rightarrow 0,
$$

причем это соотношение вьполнено равномерно по $u$ из $\left(\varepsilon N^{-1}\right)$-окрестности функции $u_{0}$.

Теорема 1 доказана.

ЗАмечАниЕ 1. Теорема 1 останется верной, если условие 1 на область $\Omega$ ослабить до следующего. Для каждой точки $x \in \partial \Omega$ и каждой окрестности $O_{1}$ точки $x$ найдется такая окрестность $O_{2} \subset O_{1}$ точки $x$, что для любой $u \in C^{m}(\bar{\Omega})$ можно указать функцию $\widetilde{u} \in C^{m}(\bar{\Omega})$ со свойствами: $u \equiv \widetilde{u}$ на $O_{2} \cap \bar{\Omega}$ и $\|\widetilde{u}\|_{C^{m}(\bar{\Omega})} \leqslant c\|u\|_{C^{m}\left(\bar{O}_{1} \cap \bar{\Omega}\right)}$, где $c$ не зависит от $u, O_{1}$ и $O_{2}$, но может зависеть от $x$ и $\Omega$.

Это ослабленное условие вьполнено для достаточно широкого класса областей. Более того, автору не известно даже, существуют ли области, для которых оно не верно. Если такие области существуют, то вопрос о справедливости для них теоремы 1 остается открытьм. 
3. Непрерывность “автономных" операторов. В этом пункте мы докажем критерий непрерьвности операторов $f$ в пространстве $C^{1}(\bar{\Omega})$ в случае, когда функция $f=f\left(\mathscr{U}_{m}\right)$ не зависит от $x$. В пространстве $C^{m}(\bar{\Omega}), m \geqslant 2$, удается получить лишь достаточное условие. Примеры показьвают, что это условие не является необходимым.

Итак, всюду в этом пункте функция $f=f\left(\mathscr{U}_{m}\right)$ не зависит от $x$.

Положим

$$
\begin{gathered}
g_{j, \varepsilon, K}(u)=\sup \left\{\left|f\left(\mathscr{U}_{m}\right)\right|:\left|u_{\alpha}\right| \leqslant \varepsilon, 1 \leqslant|\alpha|<j ; \quad\left|u_{\beta}\right| \leqslant K, j \leqslant|\beta| \leqslant m\right\}, \\
j=1, \ldots, m .
\end{gathered}
$$

ТЕОРема 2. Пусть $\Omega$ удовлетворяет условию конуса. Если для любого $K>0$ найдется такое число $\varepsilon>0$, что

$$
g_{j, \varepsilon, K} \in L_{j p+(j-1) \varepsilon, \mathrm{loc}}(\mathbb{R}), \quad j=1, \ldots, m,
$$

$u$

$$
|f(u, 0, \ldots, 0)|<\infty, \quad u \in \mathbb{R}
$$

то оператор $f$ непрерывен на $C^{m}(\bar{\Omega})$.

ДокАЗАТЕЛЬСТво. В силу теоремы 1 достаточно доказать, что в некоторой окрестности любого многочлена $p_{r}(x)$ степени $r \leqslant m$ выполнено свойство (UC). Линейной заменой переменных мы можем свести ситуацию к случаю $\partial^{r} p_{r} / \partial x_{1}^{r} \neq 0$, которьй и рассмотрим ниже. При этом мы отдельно проанализируем случаи $r=0,1$ и $r \geqslant 2$.

Если $r=0$, т.е. $p_{0} \equiv$ const, $\nabla p_{0} \equiv 0$, то непрерывность оператора $\boldsymbol{f}$ и справедливость свойства $(\mathrm{C})$ в точке $p_{0}$ сразу вытекают из непрерывности функции $f$ и условия (10).

Пусть $r \geqslant 1$. Прежде всего сведем задачу к одномерной следующим образом. Для простоты будем считать, что $\Omega=[0,1]^{n}$. Требуется оценить величину

$$
\omega_{p}(\boldsymbol{f} u, \delta)=\sup _{A \subset \Omega,|A| \leqslant \delta} \int_{A}|\boldsymbol{f} u|^{p}
$$

для $u$ из некоторой $C^{m}$-окрестности многочлена $p_{r}$. Для фиксированного множества $A \subset \Omega \mathrm{c}|A| \leqslant \delta$ рассмотрим множество $\widetilde{A}$ (определенное, вообще говоря, неоднозначно) такое, что $A \subset \widetilde{A}$ и (линейная) мера пересечения $\widetilde{A}$ с любой прямой $x_{2}=c_{2}, \ldots, x_{n}=c_{n}$ есть число, кратное $\delta$. Обозначим это число через $\mu\left(c_{2}, \ldots, c_{n}\right)$. Потребуем также, чтобы $\widetilde{A}$ имело наименьшую возможную меру, тогда $|\widetilde{A}| \leqslant|A|+\delta=2 \delta$. Введем величину

$$
\tau\left(\boldsymbol{f} u, x_{2}, \ldots, x_{n}\right)=\sup _{B \subset[0,1],|B| \leqslant \delta} \int_{B}\left|\boldsymbol{f} u\left(x_{1}, x_{2}, \ldots, x_{n}\right)\right|^{p} d x_{1} .
$$

Имеем

$$
\begin{aligned}
\int_{A}|\boldsymbol{f} u|^{p} & \leqslant \int_{\widetilde{A}}|\boldsymbol{f} u|^{p} \leqslant \frac{1}{\delta} \int_{[0,1]^{n-1}} \tau\left(\boldsymbol{f} u, x_{2}, \ldots, x_{n}\right) \mu\left(x_{2}, \ldots, x_{n}\right) d x_{2} \cdots d x_{n} \\
& \leqslant 2 \sup _{0 \leqslant x_{2}, \ldots, x_{n} \leqslant 1} \tau\left(\boldsymbol{f} u, x_{2}, \ldots, x_{n}\right) .
\end{aligned}
$$


Таким образом, чтобы получить оценку величины (11), равномерную по $u$ из некоторой окрестности $p_{r}$, достаточно получить аналогичную оценку величины $(12)$, равномерную по $u$ и $x_{2}, \ldots, x_{n}$. Так как $\partial^{r} p_{r} / \partial x_{1}^{r} \equiv$ const $\neq 0$ (пусть для определенности эта константа положительна), для $u$ из некоторой окрестности $p_{r}$ имеем $\partial^{r} u / \partial x_{1}^{r} \geqslant a>0$ и $\|u\|_{C^{m}} \leqslant K$, где $a$ и $K$ - некоторые числа.

Итак, дальше мы рассматриваем $u$ как функцию одной одномерной переменной (обозначим ее через $x$ ) и хотим получить оценку величины

$$
\tau(\boldsymbol{f} u)=\sup _{B \subset[0,1],|B| \leqslant \delta} \int_{B}|\boldsymbol{f} u(x)|^{p} d x,
$$

равномерную по функциям $u$ таким, что $\partial^{r} u / \partial x^{r} \geqslant a>0$ и $\|u\|_{C^{m}} \leqslant K$. По условию теоремы найдется такое $\varepsilon<a$, что имеет место (9). Зафиксируем его. Так как $r$-я производная функции $u$ положительна, она имеет не более $r$ участков монотонности. Достаточно рассмотреть случай, когда множество $B$, по которому идет интегрирование, целиком лежит на одном из таких участков. Тогда в (13) можем вьполнить замену переменных $x \rightarrow u(x)$ :

$$
\tau(\boldsymbol{f} u)=\sup _{B \subset[0,1],|B| \leqslant \delta} \int_{u(B)}|\boldsymbol{f} u|^{p} \frac{d u}{\left|u^{\prime}\right|} .
$$

Представим множество $B$ в виде объединения $B=\bigcup_{j=1}^{r} B_{j}$, где

$$
B_{j}=\left\{x \in(0,1):\left|u^{\prime}(x)\right| \leqslant \varepsilon, \ldots,\left|u^{(j-1)}(x)\right| \leqslant \varepsilon,\left|u^{(j)}(x)\right|>\varepsilon\right\}, \quad j=1, \ldots, r .
$$

Для $B_{1}$ имеем $\left|u^{\prime}\right|>\varepsilon$ и

$$
\int_{u\left(B_{1}\right)}|\boldsymbol{f} u|^{p} \frac{d u}{\left|u^{\prime}\right|} \leqslant \frac{1}{\varepsilon} \int_{u\left(B_{1}\right)} g_{1, \varepsilon, K}(u)^{p} d u,
$$

причем $u\left(B_{1}\right) \subset[-K, K]$ и $\left|u\left(B_{1}\right)\right| \leqslant K \delta$, так что величина (14) есть $o(1)$ при $\delta \rightarrow 0$ равномерно по $u$ из рассматриваемого множества.

На $B_{j}, j \geqslant 2$, применим неравенство Гёльдера

$$
\begin{aligned}
\int_{u\left(B_{j}\right)}|\boldsymbol{f} u|^{p} \frac{d u}{\left|u^{\prime}\right|} \leqslant & \left(\int_{-K}^{K} g_{j, \varepsilon, K}(u)^{j p+(j-1) \varepsilon} d u\right)^{p /(j p+(j-1) \varepsilon)} \\
& \times\left(\int_{u\left(B_{j}\right)} \frac{d u}{\left|u^{\prime}\right|^{j /(j-1)-\varepsilon_{j}}}\right)^{(j-1) /\left(j-\varepsilon_{j}(j-1)\right)}
\end{aligned}
$$

где $\varepsilon_{j}>0$ выбрано из условия

$$
\frac{p}{j p+(j-1) \varepsilon}+\frac{j-1}{j-\varepsilon_{j}(j-1)}=1
$$

Первьй множитель в правой части (15) конечен в силу (9) и не зависит от функции $u$ из рассматриваемого множества. Во втором интеграле вьполним обратную замену переменных $u \rightarrow x$ :

$$
\int_{u\left(B_{j}\right)}|\boldsymbol{f} u|^{p} \frac{d u}{\left|u^{\prime}\right|} \leqslant c\left(\int_{B_{j}} \frac{d x}{\left|u^{\prime}(x)\right|^{1 /(j-1)-\varepsilon_{j}}}\right)^{(j-1) /\left(j-\varepsilon_{j}(j-1)\right)} .
$$


Производная порядка $j-1$ функции $u^{\prime}$ на $B_{j}$ (т.е. функция $\left.u^{(j)}\right)$ по модулю не меньше $\varepsilon$. В силу условия $u^{(r)}>0$ функция $u^{(j)}$ имеет не более $r-j+1$ участков знакопостоянства. На каждом из этих участков индукцией по $j$ можно доказать следующее утверждение: найдутся такие многочлены $q_{1}, \ldots, q_{s}$ степени $j-1$, где $s$ зависит только от $j$, что

$$
\left|u^{\prime}(x)\right| \geqslant \min \left\{\left|q_{1}(x)\right|, \ldots,\left|q_{s}(x)\right|\right\}, \quad q_{i}(x)=\frac{\varepsilon}{(j-1) !}\left(x-x_{i}\right)^{j-1}, \quad i=1, \ldots, s .
$$

Поэтому интеграл в правой части (16) оценивается как $o(1)$ при $\left|B_{j}\right| \leqslant \delta \rightarrow 0$ равномерно по $u$ из рассматриваемого множества.

Таким образом, в некоторой окрестности любого многочлена оператор $\boldsymbol{f}$ обладает свойством (UC). Теорема доказана.

Рассмотрим несколько примеров. Прежде всего отметим, что из теоремы 2 вытекает непрерьвность оператора из примера 2 при $r<1 / p$ на $C^{1}$. Так что в этом случае теорема 2 дает точный результат. Следующий пример относится к пространству $C^{2}$.

ПРИМЕР 3. Пусть $\Omega=(0,1), r, r^{\prime}, r^{\prime \prime}>0$,

$$
f\left(x, u, u^{\prime}, u^{\prime \prime}\right)=\frac{1}{|u|^{r}+\left|u^{\prime}\right|^{r^{\prime}}+\left|u^{\prime \prime}-1\right|^{r^{\prime \prime}}} .
$$

В этом случае оператор $f$ непрерывно действует из $C^{2}$ в $L_{p}$ при $r<1 /(2 p)$ или $r^{\prime}<1 / p$ $\left(r^{\prime \prime}>0\right.$ произвольно). Теорема 2 гарантирует непрерьвность лишь при $r<1 /(2 p)$.

В оставшейся части этого пункта мы более подробно исследуем случай $m=1$. В частности, мы докажем критерии ограниченности и непрерывности оператора $f$ на $C^{1}$.

Tеорема 3. Onератор $\boldsymbol{f}: C^{1}(\bar{\Omega}) \rightarrow L_{p}(\Omega)$ ограничен тогда и только тогда, когда

$$
\left|f\left(\mathscr{U}_{1}\right)\right|<\infty \quad \text { nри всех } \quad \mathscr{U}_{1} \in \mathbb{R}^{n+1} .
$$

ДоКАЗАТЕЛЬСТВо. Напомним, что функция $f$ непрерьвна, поэтому из условия (17) очевидньм образом вытекает ограниченность оператора $f$.

Предположим, что условие (17) не вьполнено, т.е. $f\left(\mathscr{U}_{1}^{(0)}\right)=\infty$ при некотором $\mathscr{U}_{1}^{(0)}$. Рассмотрим многочлен $p_{0}$ первой степени такой, что $\left\{p_{0}(0), \nabla p_{0}(0)\right\}=\mathscr{U}_{1}^{(0)}$, и зафиксируем некоторое число $N>0$. В силу непрерывности $f$ найдется такое $\delta>0$, что

$$
\int_{[0, \delta]^{n}}\left|\boldsymbol{f} p_{0}\right|^{p}>N \delta^{n}
$$

Достаточно рассмотреть случай, когда область $\Omega$ - куб со сторонами, параллельными координатным плоскостям. Уменьшим, если нужно, $\delta$ (с сохранением условия (18)) настолько, чтобы длина ребер $\Omega$ была пропорциональна $2 \delta$. Разобьем $\Omega$ на кубы с ребрами длины $2 \delta$ и каждьй из этих кубов еще на $2^{n}$ одинаковых кубов. Из каждых $2^{n}$ маленьких кубов, составляюших один большой, выделим по одному так, чтобы выделенные кубы не имели общих (граничных) точек. На каждом из выделенных кубов определим функцию $u_{\delta}$, имеющую (с точностью до параллельного переноса) тот же график, что и $p_{0}$ на $[0, \delta]^{n}$. Методом Уитни $[8]$ продолжим $u_{\delta}$ с объединения $Q$ выделенных кубов 
на всю область $\Omega$, при этом $C^{1}$-норма функции $u_{\delta}$ увеличится не более чем в некоторое фиксированное число раз (не зависящее от $\delta$ ). Тогда

$$
\int_{\Omega}\left|\boldsymbol{f} u_{\delta}\right|^{p} \geqslant \int_{Q}\left|\boldsymbol{f} u_{\delta}\right|^{p} \geqslant \frac{|\Omega|}{(2 \delta)^{n}} \int_{[0, \delta]^{n}}\left|\boldsymbol{f} p_{0}\right|^{p}>\frac{N|\Omega|}{2^{n}} .
$$

Так как число $N$ может быть выбрано сколь угодно большим и $C^{1}$-нормы функций $u_{\delta}$ равномерно по $\delta$ ограничены, получаем неограниченность оператора $\boldsymbol{f}$.

Теорема доказана.

Из этой теоремы вытекает неограниченность оператора, рассмотренного нами в примере 2, при любых значениях параметров $r$ и $r^{\prime}$.

Tеорема 4. Пусть $m=1$. Eсли оператор $f$ действует из $C^{1}(\bar{\Omega})$ в $L_{p}(\Omega)$, то он непрерывен и выполнены условие (9), которое в данном случае принимает вид

$$
g_{K}(u)=\sup \left\{\left|f\left(\mathscr{U}_{1}\right)\right|:\left|u_{\alpha}\right| \leqslant K,|\alpha|=1\right\} \in L_{p, \text { loc }}(\mathbb{R}) \quad \forall K>0,
$$

и условие (10).

ДокАЗАТЕЛЬСТВО. В силу теоремы 2 из условий (19) и (10) вытекает непрерывность оператора $\boldsymbol{f}$. Поэтому достаточно показать, что если одно из этих условий нарушено, то найдется $u \in C^{1}(\bar{\Omega})$ такая, что $\|\boldsymbol{f} u\|_{L_{p}}=\infty$.

Если нарушено условие $(10)$, то $f\left(u_{0}, 0, \ldots, 0\right)=\infty$ для некоторого $u_{0} \in \mathbb{R}$. Но тогда для постоянной функции $u \equiv$ const $=u_{0}$ имеем $\boldsymbol{f} u \equiv \infty$.

Предположим, что условие (10) выполнено, а условие (19) нет. Тогда найдется точка $\left(u_{0}, \boldsymbol{v}_{0}\right) \in \mathbb{R} \times \mathbb{R}^{n}$ такая, что

$$
\int_{u_{0}-\varepsilon}^{u_{0}+\varepsilon} \sup _{\left|\boldsymbol{v}-\boldsymbol{v}_{0}\right|<\varepsilon}|f(u, \boldsymbol{v})|^{p} d u=\infty \quad \forall \varepsilon>0
$$

причем $\boldsymbol{v}_{0} \neq 0$ в силу (10).

Рассмотрим линейную замену переменных $\widetilde{x}=A x$, где $A$ - невырожденная матрица размера $n \times n$, первый столбец которой совпадает с $v_{0}$. Тогда $\left(u_{x_{1}}^{\prime}, \ldots, u_{x_{n}}^{\prime}\right)=\left(u_{\widetilde{x}_{1}}^{\prime}, \ldots\right.$, $\left.u_{\widetilde{x}_{n}}^{\prime}\right) A$ для любой функции $u \in C^{1}$. Введем новую функцию

$$
\widetilde{f}(u, v)=f\left(u+u_{0}, \boldsymbol{v} A\right)
$$

Функция $\tilde{f}$, очевидно, удовлетворяет условиям (10) и $(20)$ с $u_{0}=0$ и $\boldsymbol{v}_{0}=(1,0, \ldots, 0)$. Если мы докажем теорему для функции $\widetilde{f}$, то мы докажем ее и для $f$, так как операторы Немыцкого, порожденные $f$ и $\widetilde{f}$, совпадают с точностью до замены переменных в $\mathbb{R}^{n}$ и сдвига на константу в пространстве $C^{1}$.

Далее мы воспользуемся индукцией по числу переменных.

База индукции $n=1$. Положим

$$
\widetilde{g}_{\varepsilon}(u)=\sup _{|v-1|<\varepsilon}|\widetilde{f}(u, v)| .
$$


Имеем

$$
\int_{-\varepsilon}^{\varepsilon} \widetilde{g}_{\varepsilon}(u)^{p} d u=\infty
$$

при всех $\varepsilon>0$. Пусть для определенности

$$
\int_{0}^{\varepsilon} \widetilde{g}_{\varepsilon}(u)^{p} d u=\infty \quad \forall \varepsilon>0
$$

(случай $\int_{-\varepsilon}^{0} \widetilde{g}_{\varepsilon}^{p}=\infty$ рассматривается аналогично). Без ограничения общности можем считать, что $0<\widetilde{g}_{\varepsilon}(u)<\infty$ при $u \neq 0$.

Пусть $u_{j}, j=1,2, \ldots,-$ убывающая к нулю последовательность точек интервала $(0,1)$ такая, что

$$
\int_{u_{j+1}}^{u_{j}} \widetilde{g}_{1 / j}(u)^{p} d u=1
$$

В силу (21) эта последовательность содержит бесконечное число членов. Для каждой точки $\xi \in\left[u_{j+1}, u_{j}\right]$, пользуясь непрерьвностью $\widetilde{f}$, найдем такие окрестность $O_{\xi}$ и число $v_{\xi} \in[1-1 / j, 1+1 / j]$, что

$$
\left|\widetilde{f}\left(u, v_{\xi}\right)\right| \geqslant \frac{1}{2} \widetilde{g}_{1 / j}(u), \quad u \in O_{\xi} .
$$

Выделим из объединения окрестностей $O_{\xi}$ конечное подпокрытие отрезка $\left[u_{j+1}, u_{j}\right]$, скажем, $O_{j, 1}, \ldots, O_{j, l}$. Уменьшим каждую из $O_{j, i}$ так, чтобы они непересекались, но их замькания все еще покрывали отрезок $\left[u_{j+1}, u_{j}\right]$. На каждом интервале $O_{j, i}$ построим непрерьвную функцию $v_{j}(u)$, которая равна 1 на концах $O_{j, i}$ и

$$
\int_{O_{j, i}}\left|\tilde{f}\left(u, v_{j}(u)\right)\right|^{p} d u \geqslant \frac{1}{3^{p}} \int_{O_{j, i}} \tilde{g}_{1 / j}(u)^{p} d u, \quad i=1, \ldots, l .
$$

Тогда $v_{j}$ будет непрерьвной функцией на всем отрезке $\left[u_{j+1}, u_{j}\right]$, причем $v_{j}\left(u_{j+1}\right)=$ $v_{j}\left(u_{j}\right)=1$ и

$$
\int_{u_{j+1}}^{u_{j}}\left|\widetilde{f}\left(u, v_{j}(u)\right)\right|^{p} d u \geqslant \frac{1}{3^{p}}, \quad j=1,2, \ldots
$$

Функции $v_{j}$ в совокупности образуют непрерьвную функцию $v$ на $\left[0, u_{1}\right]$, если дополнительно положить $v(0)=1$. При этом

$$
\int_{0}^{\varepsilon}|\widetilde{f}(u, v(u))|^{p} d u=\infty \quad \forall \varepsilon>0
$$

Продолжим $v$ до непрерьвной функции на $\mathbb{R}$ и рассмотрим обыкновенное дифференциальное уравнение $u^{\prime}=v(u), u\left(x_{0}\right)=0$, где $x_{0} \in \Omega$. В силу известной теоремы о сушествовании решения в некоторой окрестности $O_{x_{0}}$ точки $x_{0}$ найдется функция $u(x)$ такая, что $u\left(x_{0}\right)=0, u^{\prime}\left(x_{0}\right)=v(0)=1$ и $1 / 2 \leqslant u^{\prime}(x)=v(u(x)) \leqslant 2$, т.е. $u$ монотонно возрастает и

$$
\int_{O_{x_{0}}}\left|\tilde{f}\left(u(x), u^{\prime}(x)\right)\right|^{p} d x=\int_{u\left(O_{x_{0}}\right)}|\tilde{f}(u, v(u))|^{p} \frac{d u}{v(u)} \geqslant \frac{1}{2} \int_{u\left(O_{x_{0}}\right)}|\tilde{f}(u, v(u))|^{p} d u=\infty
$$


Продолжая $u$ с $O_{x_{0}}$ до функции из $C^{1}(\bar{\Omega})$, завершаем доказательство в случае $n=1$. Отметим, что функция $u$ может быть выбрана сколь угодно близкой к многочлену $p(x)=$ $x-x_{0}$.

Шаг индукции. Предположим, что утверждение верно для функций $n-1$ переменных, т.е. из $(20)$ с $\boldsymbol{v} \in \mathbb{R}^{n-1}$ вытекает существование такой функции $u\left(x_{1}, \ldots, x_{n-1}\right)$, что $\|\boldsymbol{f} u\|_{L_{p}}=\infty$. При этом в $(20) u_{0}=0, \boldsymbol{v}_{0}=(1,0, \ldots, 0)$ и упомянутая функция $u$ может быть выбрана сколь угодно близко к многочлену $p(x)=x_{1}-x_{1}^{(0)}$, где $x_{1}^{(0)}$ - первая координата некоторой фиксированной точки $x^{(0)} \in \Omega$.

Достаточно рассмотреть случай, когда область $\Omega$ имеет вид куба $(0,1)^{n}$.

Положим $\widetilde{g}_{\varepsilon}\left(u, v_{1}, \ldots, v_{n-1}\right)=\sup _{\left|v_{n}\right|<\varepsilon}|\widetilde{f}(u, v)|$. Тогда к $\widetilde{g}_{\varepsilon}$ применимо предположение индукции и найдется функция $u$, определенная на $[0,1]^{n-1}$, такая, что

$$
\int_{[0,1]^{n-1}} \widetilde{g}_{\varepsilon}\left(u, u_{x_{1}}^{\prime}, \ldots, u_{x_{n-1}}^{\prime}\right)^{p} d x_{1} \cdots d x_{n-1}=\infty
$$

Рассуждая, как и выше, строим непрерывную функцию $v\left(x_{1}, \ldots, x_{n-1}\right),|v|<\varepsilon$, для которой

$$
\int_{[0,1]^{n-1}} \tilde{f}\left(u, u_{x_{1}}^{\prime}, \ldots, u_{x_{n-1}}^{\prime}, v\right)^{p} d x_{1} \cdots d x_{n-1}=\infty .
$$

Мы можем считать, что функция $u$ задана на стороне $[0,1]^{n-1} \times\{0\}$ куба $\Omega$. Продолжим ее на $\mathbb{R}^{n}$ (например, методом Уитни [8]) так, чтобы $u_{x_{n}}^{\prime}=v$ при $x_{n}=0$, и затем возьмем сужение на параллелепипед $\Omega_{\delta}=[0,1]^{n-1} \times[0, \delta]$. Теперь снова продолжим ее на $\Omega_{1 / 2}$ так, чтобы она имела период $2 \delta$ по $x_{n}$. Выбирая $\delta$ достаточно малым, мы можем сделать сколь угодно большой $L_{p}$-норму функции $\boldsymbol{f} u$ на $\Omega_{1 / 2}$, при этом $C^{1}$-норма функции $u$ будет ограничена константой, не зависящей от $\delta$. Обозначим эту функцию $u_{1}$.

Аналогично строим функции $u_{j}$ на $\Omega_{1-1 / 2^{j}} \backslash \Omega_{1-1 / 2^{j-1}}$ с условиями $\left(p(x)=x_{1}\right.$, $j=2,3, \ldots)$

$$
\begin{gathered}
\left\|u_{j}-p\right\|_{C^{1}\left(\Omega_{1-1 / 2^{j}} \backslash \Omega_{1-1 / 2^{j-1}}\right)} \leqslant \frac{1}{4^{j}}, \\
\int_{\Omega_{1-1 / 2^{j}} \backslash \Omega_{1-1 / 2^{j-1}}}\left|\boldsymbol{f} u_{j}\right|^{p} \geqslant 1 .
\end{gathered}
$$

Немного изменив определение функций $u_{j}$, можно добиться их гладкой стьковки. Таким образом, опуская детали, получаем искомую $C^{1}$-функцию $u$ на $\bar{\Omega}$ :

$$
\begin{gathered}
u=u_{j} \quad \text { на } \Omega_{1-1 / 2^{j}} \backslash \Omega_{1-1 / 2^{j-1}}, \quad j=1,2, \ldots \\
\int_{\Omega}|\boldsymbol{f} u|^{p}=\sum_{j=1}^{\infty} \int_{\Omega_{1-1 / 2} \backslash \Omega_{1-1 / 2^{j-1}}}\left|\boldsymbol{f} u_{j}\right|^{p}=\infty
\end{gathered}
$$

Отметим, что условие (23) гарантирует существование первых производных функции $u$ в точках с $x_{n}=1$ и их непрерьвность. Теорема доказана.

Из теорем 2, 4 вытекает 
СлЕДСТВИЕ 2. Пусть $\Omega$ удовлетворяет условию конуса. Oператор $f: C^{1}(\bar{\Omega}) \rightarrow$ $L_{p}(\Omega)$ непрерывен тогда и только тогда, когда выполнены условия (19) $и$ (10).

Приложения. Используя те же идеи, что и при доказательстве теоремы 2 , мы можем получить, например, следующий признак непрерывности оператора $\boldsymbol{f}$.

Tеорема 5. Пусть $x_{0} \in \bar{\Omega}, M=\#\{\alpha:|\alpha| \leqslant m\}$,

$$
\left|f\left(x, \mathscr{U}_{m}\right)\right| \leqslant a(x) b\left(\mathscr{U}_{m}\right), \quad x \in \Omega, \quad \mathscr{U}_{m} \in \mathbb{R}^{M},
$$

где $a \in L_{p, \operatorname{loc}}\left(\bar{\Omega} \backslash\left\{x_{0}\right\}\right)$ и $b$ - непрерывная конечнозначная функиия. Для произвольного многочлена $q(x)$ степени $m$ и $K>0$ положим

$$
\begin{gathered}
g_{K}(x, q)=\sup \left\{\left|f\left(x, \mathscr{U}_{m}\right)\right|: D^{\alpha} q(x)-K\left|x-x_{0}\right|^{m-\alpha}<u_{\alpha}<D^{\alpha} q(x)+K\left|x-x_{0}\right|^{m-\alpha},\right. \\
|\alpha| \leqslant m\} .
\end{gathered}
$$

Если для любого $K>0$

$$
\sup _{\|q\|_{C} \leqslant K} \int_{\left|x-x_{0}\right|<\varepsilon} g_{K}(x, q)^{p} d x=o(1), \quad \varepsilon \rightarrow 0,
$$

то оператор $f: C^{m}(\bar{\Omega}) \rightarrow L_{p}(\Omega)$ непрерывен.

ПРИМЕР 4. Положим $\Omega=(0,1)$,

$$
f(x, u)=\ln \left[e^{-1 / x}+\left(u^{2}-x\right)^{2}\right] .
$$

Чтобы показать, что этот оператор со значениями в $L_{1}$ непрерывен на пространстве $C^{1}(\bar{\Omega})$, воспользуемся теоремой 5 с $x_{0}=0, a(x)=1 / x, b(u)=\max \left\{1, \ln \left[1+\left(u^{2}+1\right)^{2}\right]\right\}$. При этом

$$
g_{K}(x, k x+r) \leqslant 2|\ln |(k x+r+\theta(x) x)^{2}-x||+c, \quad-K \leqslant \theta(x) \leqslant K .
$$

Нетрудно показать, что верхняя грань в (24) достигается, если $|\theta(x)| \equiv K$ в некоторой окрестности нуля. С учетом этого замечания легко получить доказательство самой оценки (24).

В качестве приложения полученных результатов приведем следующее утверждение о непрерьвности операторов Немыцкого на пространствах Соболева $W_{p}^{m}(\Omega)$.

Tеорема 6. Пусть $1<p<\infty, 1 \leqslant q<\infty, m>0, p^{\prime}=p /(p-1)$, область $\Omega$ удовлетворяет условию конуса и имеет место оченка

$$
\left|f\left(x, \mathscr{U}_{m}\right)\right| \leqslant C_{0}\left(x, \mathscr{U}_{m_{0}}\right)+C_{1}\left(\mathscr{U}_{m_{0}}\right) \sum_{m_{0}<|\alpha| \leqslant m} u_{\alpha}^{r_{\alpha} / q},
$$

где $m_{0}$ - максимальное челое число, строго меньшее $m-n / p$,

$$
\begin{array}{ll}
r_{\alpha}=\frac{n p}{n-(m-|\alpha|) p} & \text { при } m-\frac{n}{p}<|\alpha| \leqslant m, \\
r_{\alpha}-\text { любое положительное число } & \text { при }|\alpha|=m-\frac{n}{p},
\end{array}
$$

$C_{1}: \mathbb{R}^{M} \rightarrow \mathbb{R}$ - непрерывная функиия, $M=\#\left\{\alpha:|\alpha| \leqslant m_{0}\right\}$, а $C_{0}$ мохет быть представлено в виде суммы функиий (зависящих от тех же переменных, что $\left.C_{0}\right)$, каждая из которых удовлетворяет условию одной из теорем 1,2 или 5 непрерывности на пространстве $C^{m_{0}}(\bar{\Omega})$.

Тогда оператор Немыцкого $f$, порожденный функиией $f$, непрерывно действует из $W_{p}^{m}(\Omega)$ в $L_{q}(\Omega)$. 
ДокАЗАТЕльство непосредственно вытекает из теорем вложения Соболева с учетом теорем A, $1,2,5$.

Отметим, что аналогичное утверждение справедливо, конечно, и для пространств с дробным показателем гладкости.

\section{СПИСОК ЦИТИРОВАННОЙ ЛИТЕРАТУРЫ}

[1] Красносельский М. А. Топологические методы в теории нелинейных интегральных уравнений. М.: ГИТТЛ, 1956.

[2] Вайнберг М. М. Вариационные методы исследования нелинейных операторов. М.: Гостехиздат, 1956.

[3] Шрагин И. В. О непрерьвности оператора Немьщкого // Функцион. анализ и его применение. Труды V Всесоюзной конференции по функциональному анализу и его применению. Баку: Изд-во АН АзССР, 1961. Р. 272-277.

[4] Chiappinelli R., Nugari R. The Nemitskii operator in Hölder spaces: some necessary and sufficient conditions // J. London Math. Soc. (2). 1995. V. 51. № 2. P. 365-372.

[5] Sickel W. Composition operators acting on Sobolev spaces of fractional order-a survey on sufficient and necessary conditions // Function Spaces, Differential Operators, and Nonlinear Analysis (Paseky nad Jizerou, 1995). Prague: Prometheus, 1996. P. 159-182.

[6] Appell J., Zabrejko P. P. Nonlinear Superposition Operators. Cambridge: Cambridge Univ. Press, 1990.

[7] Бесов О. В., Ильин В. П., Никольский С. М. Интегральные представления функций и теоремы вложения. М.: Наука, 1996.

[8] Whitney H. Analytic extensions of differentiable functions defined in closed sets // Trans. Amer. Math. Soc. 1934. V. 36. P. 63-89. 